\begin{tabular}{ccc}
\hline & International Journal of Aging Research \\
(ISSN:2637-3742)
\end{tabular}

\title{
Diagnosis and monitoring of Alzheimer Disease with saliva biomarker BACE1
}

\section{Dr SJ Kellner and Prof M Ferchichi}

Mesencell Biotech International Ltd, 20-22 Wenlock Road, London, N1 7GU, United Kingdom

\section{ABSTRACT}

Alzheimer's disease (AD) is characterized by the slow decline of cognition and functional abilities over time. The diagnosis for probable and possible AD relies principally on clinical criteria. The confirmation of the disease is made post-mortem by identifying extracellular senile plaques and intraneuronal fibrillary tangles in the brains of subjects with clinically defined dementia. However, the field critically lacks validated AD specific peripheral biomarkers to support the diagnosis in living patients or for early detection of patients at risk before symptoms appear. BACE1 (Beta site amyloid precursor protein cleaving enzyme 1) cleaves Amyloid Precursor Protein (APP) at two beta sites and represents a key target enzyme in the monitoring and possible treatment of $A D$. In our preliminary study, we evaluated the usefulness of salivary BACE1 to determine risk to develop $A D$ in clinically normal patients. A preliminary in house laboratory grading system for BACE1 content in saliva was established with high levels of salivary BACE1 present in older patients and putatively at risk to develop AD. BACE1 seems to be a useful biomarker to help diagnose $A D$ and to monitor disease progression, and remarkably regression, when treated with stem cell secretome.

\section{Keywords:}

Biomarkers, Alzheimer Disease, Mesenchymal stem cells, amyloid beta, amyloid precursor protein, amyloid plaques, BACE1, neprilysin, micro RNA, UHC-L1, disease monitoring, saliva
${ }^{*}$ Correspondence to Author:

Dr SJ Kellner and Prof M Ferchichi Mesencell Biotech International Ltd, 20-22 Wenlock Road, London, N1 7GU, United Kingdom

How to cite this article:

SJ Kellner and M Ferchichi. Diagnosis and monitoring of Alzhei mer Disease with saliva biomarker BACE1. International Journal of Ag ing Research, 2018, 1:21

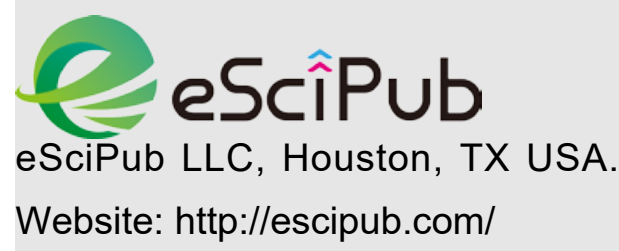




\section{Introduction}

Alzheimer's disease (AD) is characterized by the slow decline of cognition and functional abilities over time. The diagnosis for probable and possible $A D$ relies principally on clinical criteria and neuropsychological tests defined by the National Institute of Neurological Disorders and Stroke (NINDS) and the Alzheimer's disease and Related Disorders Association (ADRDA) (1), mostly by assessment through a Mini-Mental State Examination MMSE. The confirmation of the disease is made postmortem by identifying extracellular senile plaques and intraneuronal fibrillary tangles in the brains of subjects with clinically defined dementia. However, the field critically lacks validated $A D$ specific peripheral biomarkers to support the diagnosis in living patients or for early detection of patients at risk before symptoms appear. Some investigators studied structural Magnetic Resonance Imaging (2) as means for in vivo confirmation, whereas others concentrate on liquid biomarkers, such as plasma amyloid beta $(A \beta)-40$ and 42 , albeit with mixed results (3).

$A D$ is the most common form of dementia and is pathologically characterized by amyloid plaques, neurofibrillary tangles and loss of synapses in the brain. Neurofibrillary tangles consist of hyperphosphorylated tau protein while fibrils of the amyloid beta-peptide $(A b)$ aggregate into amyloid plaques (4). Smaller, oligomeric species of $A b$ have proven to be neurotoxic and cause synaptic dysfunction (5). Ab is a proteolytic product of the amyloid precursor protein (APP) which is sequentially cleaved in an amyloidogenic pathway by beta- and gamma-secretases (4). Beta-secretase cleaves APP close to the membrane, releasing a soluble APP beta-fragment (sAPPb). The remaining Cterminal fragment (APP-CTFb) can then be cleaved by gamma-secretase at different sites yielding $A b$-peptides of varying length, $A b 40$ being the most common, while Ab42 is more toxic and prone to aggregate. If APP is cleaved in a non-amyloidogenic pathway by alpha- secretase instead of beta-secretase, a non-toxic P3 fragment will be formed after the gammasecretase cleavage.

Beta-site APP-cleaving enzyme 1 (BACE1), the beta-secretase in vivo, has been identified as a 501-amino acid residue single transmembrane aspartyl protease (6). BACE1 cleaves APP at two beta sites and produces the C-terminal fragments (CTF) C99 and C89 (7). C99 is subsequently cleaved by presenilin (PS)dependent gamma-secretase complex to release $A b$ fragments. BACE1 therefore represents a key target enzyme in the monitoring and possible treatment of $A D$. However, over the last decade, it has become clear that BACE1 proteolytically cleaves a number of substrates in addition to APP. When experimenting with total knock out BACE1 mice, the extreme reduction or total absence of BACE1 led to neurological dysfunctions (8). Protocols using mesenchymal stem cells and/or their cell-free secretions (secretome) did not show these disadvantages, as, although many active ingredients are present and have shown to be beneficial, the concentration of these ingredients, the diverse mode of actions (i.e. anti-inflammatory molecules, content of alpha secretase neprilysin (NEP), antioxidants and ubiquitin carboxyl-terminal hydrolase L1) and long term but temporary cellular activation via microRNA prevent drastic changes. That being said, the content of amyloid beta degrading NEP and ubiquitin carboxyl-terminal hydrolase L1 (UCH-L1) in the secretome of mesenchymal stem cells (MSCs), have been investigated in some detail in preclinical in vitro and in vivo studies (9). Also, on the production side of the secretome, both ingredients can be augmented through GMP compatible manufacturing processes. UCH-L1 accelerates BACE1 degradation and affects APP processing and $A b$ production (10).

In our preliminary study, we evaluated the usefulness of salivary BACE1 to determine risk to develop AD in clinically normal patients.

\section{Methods and Material}

IJOAR: http://escipub.com/international-journal-of-aging-research/ 


\section{Salivary samples}

Salivary samples of 8 Patients were taken first thing in the morning, at lunchtime and evening before eating and drinking. Sample size was approximately $1 \mathrm{ml}$. $200 \mathrm{ul}$ were mixed with same amount of protease inhibitor (Protease Inhibitor Cocktail, Sigma Aldrich, Switzerland). Samples with and without protease inhibition were either frozen immediately or left for 48 and 72 hours at room temperature before freezing them as well at minus 20 degrees Celsius before analyzing.

Saliva samples were not centrifuged or otherwise processed.

\section{ELISA}

Human Beta site APP cleaving enzyme 1 sandwich ELISA kit (Sun Long Biotech, China) was used. $10 \mathrm{ul}$ salivary samples and diluted salivary samples (1:1 with protease inhibitor) were processed according to standard procedure, measured at $450 \mathrm{OD}$ and BACE 1 concentration in $\mathrm{pg} / \mathrm{ml}$ calculated with the help of the standard curve.

\section{Patients}

Patients were all healthy with no clinical signs of Alzheimer disease. Young patients were not showing any signs of mental deficiencies but some of the older Patients were self-assessed as having problems with concentration and memory. There were no family incidences of Alzheimer Disease.

\section{MMSE}

A German version of the mini-mental state examination was performed.

\section{Results}

\section{Mini-Mental State Examination}

MMSE was normal in all patients. However, two patients had self-assessed problems with concentration and memory, which the MMSE did not pick up.

\section{Saliva samples}

Saliva samples from morning, midday and evenings were analyzed (Table 1). In all samples BACE1 could be detected. There was no clear diurnal variation, but clear differences in samples in 7 out of 8 Patients regarding the time of sampling. Levels of BACE 1 varied from $267 \mathrm{pg} / \mathrm{ml}$ to $939 \mathrm{pg} / \mathrm{ml}$.

Saliva samples of three Patients were studies to see if BACE1 content at room temperature would change over 72 hours (Table 2). Fresh samples had a lower content of BACE1 then when measured after 48 respectively 72 hours.

Fresh saliva samples were compared with samples stabilized with protease inhibitors (Table 3). Results showed a big difference between the two groups.

Table 1: Diurnal variations to BACE 1 levels in saliva

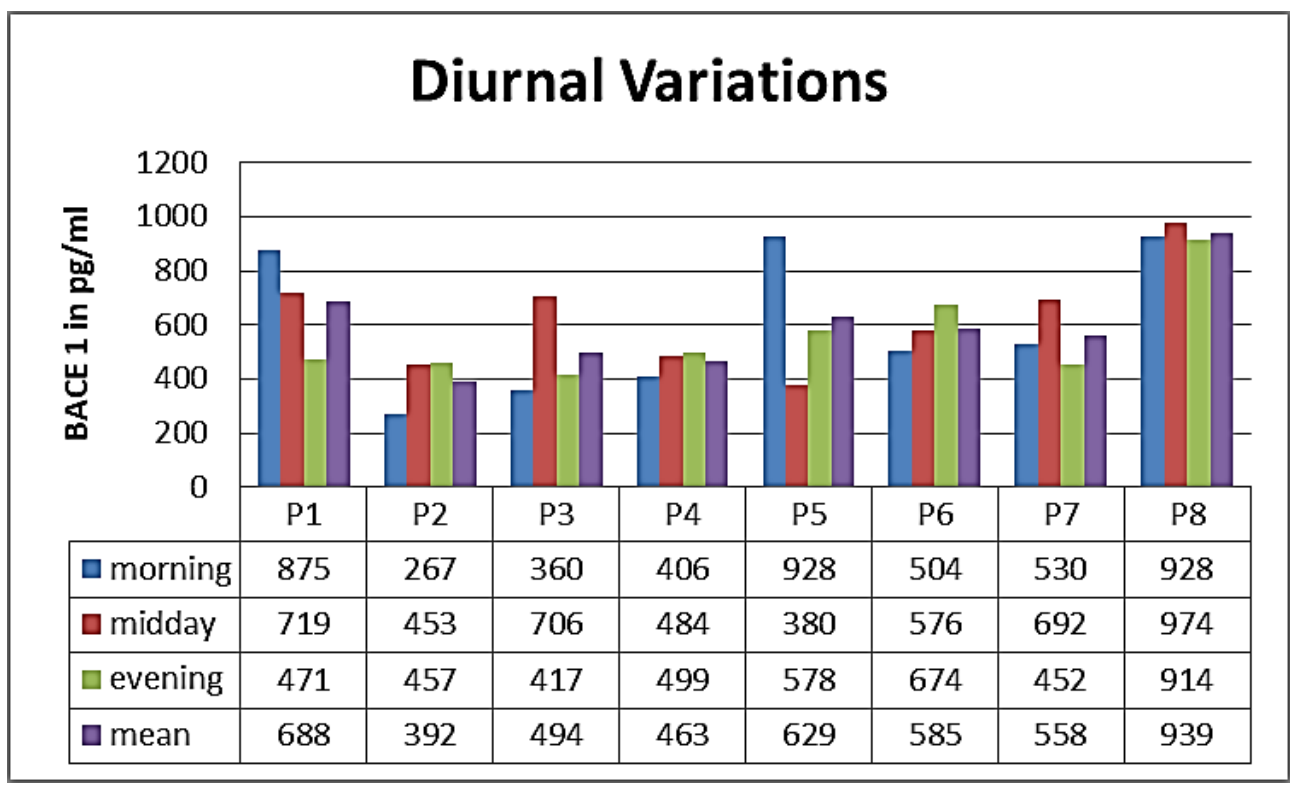

IJOAR: http://escipub.com/international-journal-of-aging-research/ 
Table 2: BACE1 values in saliva according to time spent before analysis

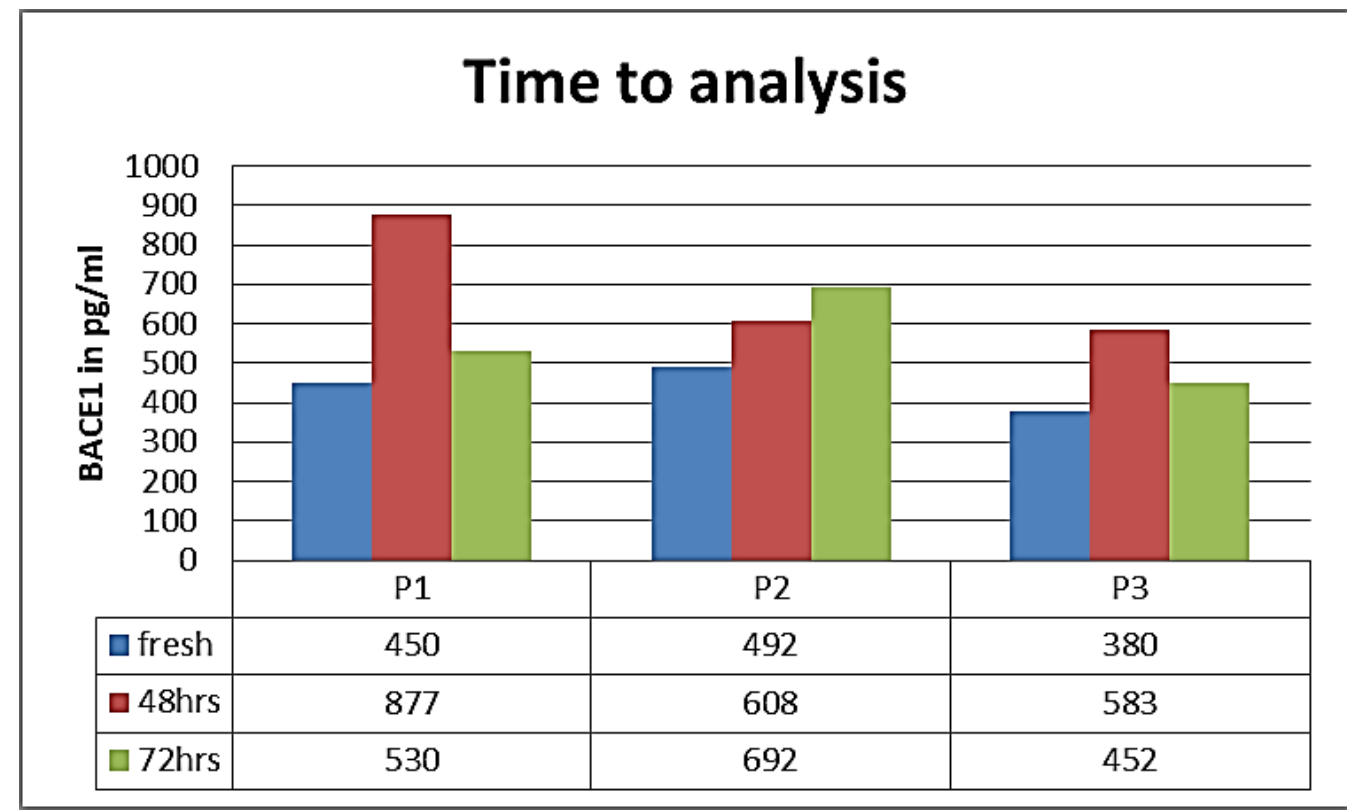

Table 3: Influence of protease inhibitor on BACE1 content in saliva samples

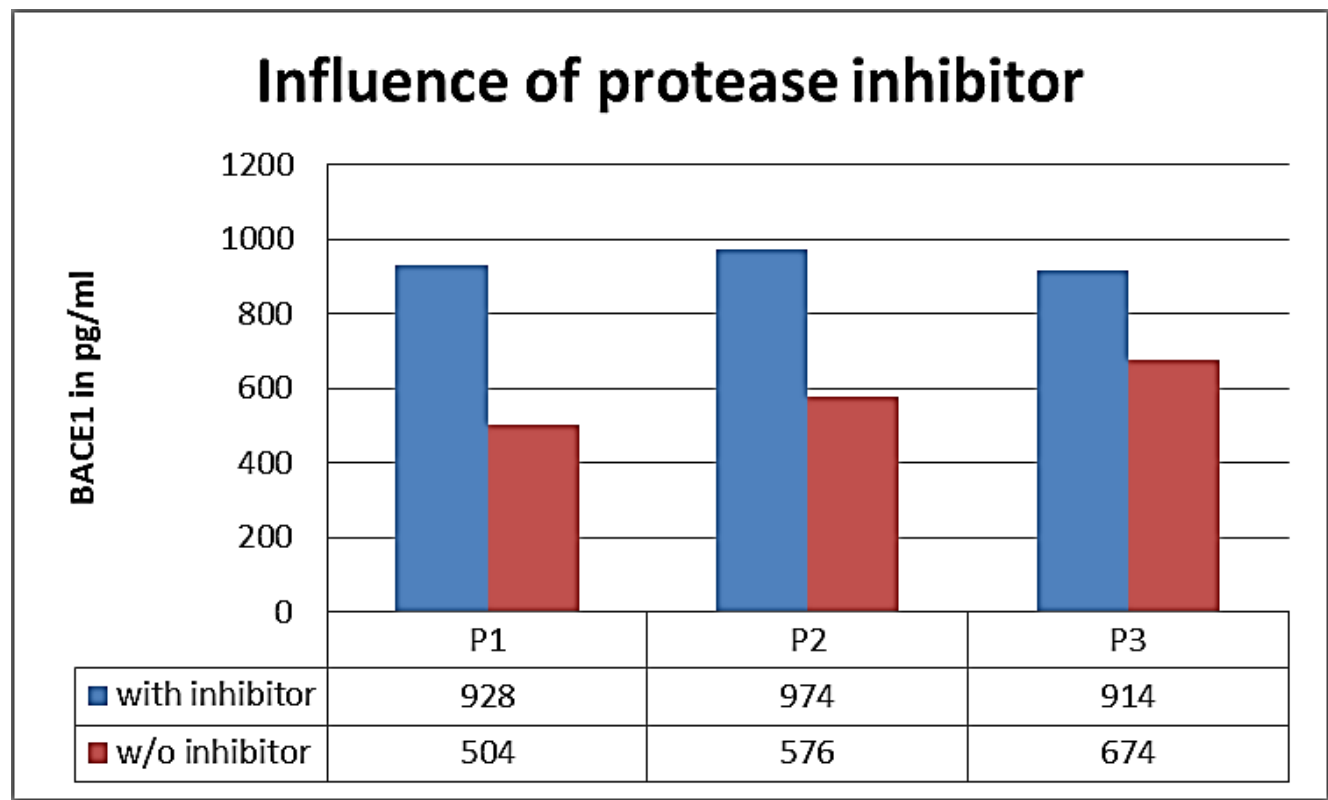

\section{ELISA}

ELISA tests were straight forward; however the small sample size of $10 \mathrm{ul}$ and the viscosity of saliva made it difficult to accurately and consistently dose the wells.

\section{Patients}

Our 8 patients were aged between 18 years of age and 62 years of age. The upper limit was meant to prevent including non-diagnosed early stages AD patients. There were 3 male and 5 female Patients. 2 patients, both female, were suspected to have a certain degree of memory problems without any influence on their MMSE.

\section{Discussion}

Saliva samples are easily obtained, but variation in the amount of saliva is well known, with $A D$ patients having more saliva production then normal controls. Theoretically, the total amount of saliva production and a weight factor would be needed for accuracy. Practically, although asked to be diligent, patients had quite a few variations in the time of sampling, making this in AD patients a difficult task. Time of delay between sampling and processing the samples revealed remarkable differences. The initial idea was that BACE1 would decline with passing 
hours before processing, which was not proven to be true. All the tested samples showed an increase of BACE1 values in the ELISA during the delay, however not linearly. This could be due to BACE1 not being freely suspended in saliva. Studies on animals have shown that within the brain, BACE1 is enriched in synaptic vesicles (11). Protease inhibition resulted consistently in higher levels of BACE1 being preserved in saliva. As with all tests, saliva sampling should follow a concise and practical protocol, allowing comparison within the same laboratory and patient setting. We established a protocol consisting of fasting saliva sampling before breakfast, with saliva allowed to pool in the anterior part of the mouth behind the front teeth with the mouth held open for 30 to 60 seconds. Saliva was removed with flexible plastic non-sterile plastic pipettes and transferred to $1.5 \mathrm{ml}$ plastic sample vials and stored on ice immediately. Vials with the saliva sample then should be frozen within 1 hour. No anti-protease was added, as early saliva samples could not be pipetted accurately due to foaming.

\section{Table 4: proposed Cut-off values for BACE1 saliva values}

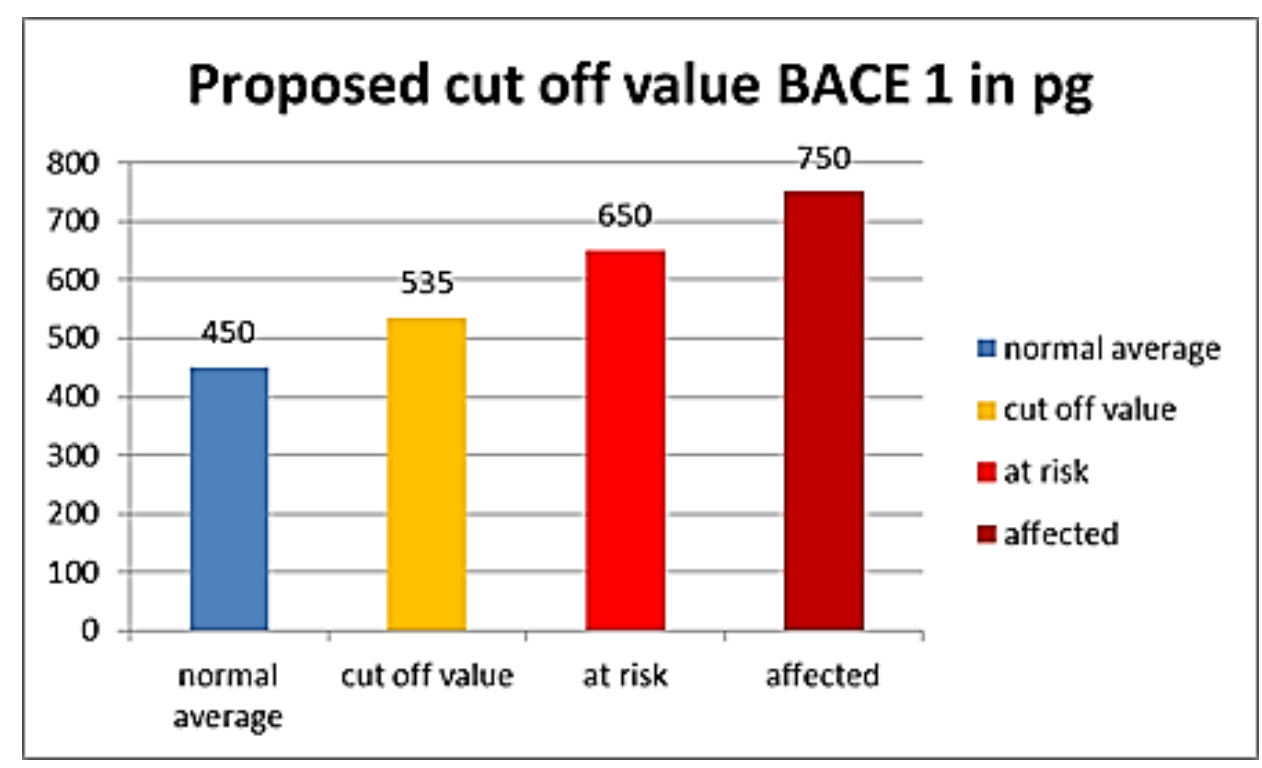

All salivary samples tested revealed content of BACE 1 biomarker. Content of BACE1 varied considerably during daytime and with selfsampling by the patients. In a separate study, this variability could be lessened by our sampling protocol and by doing the sampling by a nurse or technician. Contrary to blood as biomarker, saliva reacts quite closely to changes in CNS. BACE1 is certainly implicated in the $A D$ process and thus our results with elevated BACE1 saliva levels associated with $A D$ (separate, not published to date) and with beginning cognitive impairment in our group of patients confirm the value of saliva as biomarker as well as the connection between elevated BACE1 levels in saliva with cognitive impairment and AD. This will have to be confirmed with a bigger number of patients, but is in itself logical and plausible. Other researchers (3) have found that BACE1 levels in blood platelets are lowered (sic!) in $A D$ patients, which does just not seem logical.

Based on these test we have set a cut off value for BACE1 saliva content in AD patients, patients at risk and normal patients (Table 4). These values should be considered laboratory specific and should be validated freshly when used in another setting. We also believe that we can predict $A D$ or at least a higher risk to develop $A D$ at a very early time, giving hope to prevent further accumulation of amyloid aggregates and even clear existing amyloid 
aggregates with stem cell therapy.

Two of our patients had stem cell secretome treatments before and after saliva sampling. The secretome is a patented, cell free product generated from the active ingredients which stem cells secrete as cell to cell communication or as a result of being stressed by various degrees of hypoxia or by preconditioning of the stem cell media before the production of the secretome. The secretome can thus be adapted to different diseases, as we have shown in other studies, i.e. beneficial for cartilage regeneration or with more content of neuroregenerative substances. One of the patients was in the group of at risk patients to develop AD and one patient had normal saliva values of BACE1. They both received intramuscular secretome 4 times at intervals of 2-4 weeks. Salivary BACE1 levels sank dramatically in both patients. This confirms the treatment potential of AD with the secretome, although it is not clear which of the ingredients play a major role in this. As a total BACE1 knockout in rodents also lead to neurological dysfunction (8), a total block of BACE1 does not seem beneficial. However, the significant lowering of BACE1 over a long period of time would at least prevent new formation of amyloid beta aggregates and give to body a chance to clear aggregated proteins via the ubiquitin protease system. UCH-L1 in the secretome not only inhibits neuritic plaque formation, but also accelerates degradation of BACE1, regulates APP and is a major component of the ubiquitin protease system (12, 13).

It is still early days, and we are expanding our stem cell preventative treatments to the treatment of clinically manifest cognitive impairment and Alzheimer disease. The saliva biomarker BACE1 will give us a tool to monitor disease progression or regression, before clinical signs become evident.

\section{References}

1. McKhann GM, Knopman DS, Chertkow H, Hyman BT, Jack CR Jr, Kawas CH, Klunk WE, Koroshetz WJ, Manly JJ, Mayeux R, et al. The diagnosis of dementia due to Alzheimer's disease:

recommendations from the National Institute on Aging-Alzheimer's Association workgroups on diagnostic guidelines for Alzheimer's disease. Alzheimers Dement. 2011; 7:263-269. [PubMed: 21514250]

2. Yanhong Zhou, Chuangchuang Tan, Dong Wen, Hongmin Sun, Wei Han and Yuchen Xu: The Biomarkers for Identifying Preclinical Alzheimer's Disease via Structural and Functional Magnetic. Front. Aging Neurosci., 27 April 2016 https://doi.org/10.3389/fnagi.2016.00092

3. Koyama A, Okereke OI, Yang T, Blacker D, Selkoe DJ, Grodstein F. Plasma Amyloid-beta as a Predictor of Dementia and Cognitive Decline: A Systematic Review and Meta-analysis. Arch Neurol.2012

4. Selkoe D. J. (2011) Alzheimer's Disease. Cold Spring Harb. Perspect. Biol. 3, a004457

5. Bayer T. A. and Wirths O. (2010) Intracellular accumulation of amyloidBeta - a predictor for synaptic dysfunction and neuron loss in Alzheimer's disease. Front. Aging Neurosci. 2, 8.

6. Roberds S. L., Anderson J. and Basi G. et al. (2001) BACE knockout mice are healthy despite lacking the primary beta-secretase activity in brain: implications for Alzheimer's disease therapeutics. Hum. Mol. Genet. 10, 1317-1324.

7. Sun X., He G. and Song W. (2006a) BACE2, as a novel APP thetasecretase, is not responsible for the pathogenesis of Alzheimer's disease in Down syndrome. FASEB J. 20, 1369-1376.

8. Kathryn M. Munro, Amelia Nash, Martina Pigoni, Stefan F. Lichtenthaler \& Jenny M. Gunnersen. Functions of the Alzheimer's Disease Protease BACE1 at the Synapse in the Central Nervous System. J Mol Neurosci (2016) 60:305-315

9. Latha Devi and Masuo Ohnu. A combination Alzheimer's therapy targeting BACE1 and Neprilysin in 5XFAD transgenic mice. Molecular Brain (2015) 8:19

10. Mingming Zhang et al..Control of BACE1 degradation and APP processing by ubiquitin carboxyl-terminal hydrolase L1. Journal of Neurochemistry, 2012, 120:1129-1138

11. Lundgren et al.. ADAM10 and BACE1 are localized to synaptic vesicles. Journal of Neurochemistry 2015, 135, 606-615

12. Tramutola et al., 2016: It is all about Ubiquitin: Role of altered Ubiquitin-Proteasome System and UCHL1 in Alzheimer Disease. Oxidative Medicine and Longevity Vol 2016, ArtID2756068

13. Gong et al., 2016: The Ubiquitin-Proteasome System: Potential Therapeutic Targets for Alzheimer Disease and Spinal Cord Injury. Front.Mol.Neurosci.9:4

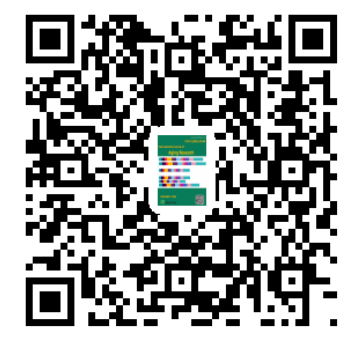

IJOAR: http://escipub.com/international-journal-of-aging-research/ 